\title{
Distinctive Features of Adult Ocular Dominance Plasticity
}

\author{
Masaaki Sato and Michael P. Stryker \\ Department of Physiology, W. M. Keck Foundation Center for Integrative Neuroscience, University of California, San Francisco, San Francisco, California \\ 94143-0444
}

\begin{abstract}
Sensory experience profoundly shapes neural circuitry of juvenile brain. Although the visual cortex of adult rodents retains a capacity for plasticity in response to monocular visual deprivation, the nature of this plasticity and the neural circuit changes that accompany it remain enigmatic. Here, we investigate differences between adult and juvenile ocular dominance plasticity using Fourier optical imaging of intrinsic signals in mouse visual cortex. This comparison reveals that adult plasticity takes longer than in the juvenile mouse, is of smaller magnitude, has a greater contribution from the increase in response to the open eye, and has less effect on the hemisphere ipsilateral to the deprived eye. Binocular deprivation also causes different changes in the adult. Adult plasticity is similar to juvenile plasticity in its dependence on signaling through NMDA receptors. We propose that adult ocular dominance plasticity arises from compensatory mechanisms that counterbalance the loss of afferent activity caused by visual deprivation.
\end{abstract}

Key words: visual cortex; critical period; mouse; optical imaging; vision; plasticity

\section{Introduction}

The remarkable behavioral adaptability to altered sensory environments is one of the most prominent features of the brain. Normal sensory experience during an animal's early life is required for establishment and maintenance of proper function of its sensory systems, and sensory deprivation in early life can exert persistent and devastating effects (Jacobson et al., 1981). Responses of neurons in the visual cortex to the two eyes provide a dramatic example of experience-dependent plasticity of a sensory cortical circuit, one that has been studied over more than four decades (Wiesel, 1982). In ocular dominance (OD) plasticity, brief deprivation of patterned vision in one eye leads to a reduction in the responses of visual cortical neurons to the deprived eye and a matching increase in responses to the open eye. Sensitivity to this manipulation is high in juvenile animals during a critical period in early life, after which the cortical neurons become less sensitive to monocular deprivation (MD).

There is also plasticity in adult sensory cortex, including the visual cortex (Buonomano and Merzenich, 1998; Fox and Wong, 2005). An understanding of cortical plasticity in the adult brain is important because it has the potential to provide a rational basis for therapy. The same manipulations that induce plasticity in the critical period are reported to have similar, but somewhat slower effects in the adult (Sawtell et al., 2003; Pham et al., 2004). However, near the close of the critical period, there are dramatic differences in the anatomical rearrangements and laminar-specific

Received May 30, 2008; revised Aug. 8, 2008; accepted Aug. 24, 2008

This work was supported by grants from the National Institutes of Health to M.P.S. M.S. is a recipient of Uehara Memorial Foundation and the Japan Society for the Promotion of Science Fellowships. We are grateful to Professors Juan Korenbrot, David Sretavan, and Matthew LaVail for sharing their equipment and expertise, and to Maha Abdulla for helping with behavioral experiments.

Correspondence should be addressed to Michael P. Stryker, Department of Physiology, University of California, San Francisco, 513 Parnassus Avenue, Room HSE-802, San Francisco, CA 94143-0444. E-mail: stryker@phy.ucsf.edu. DOI:10.1523/JNEUROSCI.2451-08.2008

Copyright $\odot 2008$ Society for Neuroscience ～0270-6474/08/2810278-09\$15.00/0 physiological changes produced by MD that begins at different ages (LeVay et al., 1980; Daw et al., 1992). These studies would suggest that the neural circuits and plasticity mechanisms in primary visual cortex are not affected identically by MD performed in adulthood and during the critical period.

Here, we compare the plasticity induced in mouse visual cortex by monocular and binocular deprivation during the critical period with plasticity during adulthood. We sought to determine whether the plasticity at different ages was similar in character and differed only in rate or extent, or whether there were also qualitative differences. We hoped that such a side-by-side comparison would reveal differences if they existed. We used optical imaging of the intrinsic signal of cortical activity for our measurements of visual responses to stimulation through the two eyes because it provides quantitative unbiased estimates of cortical responses that are not affected by the modest sample of single neurons from which conclusions about ocular dominance have conventionally been drawn.

Our results reveal clear qualitative differences between critical period and adult plasticity in mouse visual cortex. It will be important for future work to elucidate the specific cellular mechanisms and neural circuits that retain the capacity for plasticity in the adult.

\section{Materials and Methods}

All experiments were conducted in accordance with protocols approved by the University of California, San Francisco Institutional Animal Care and Use Committee.

Mouse surgery. For monocular and binocular deprivation, one or both eyelids of C57BL/6 mice (Simonsen Laboratories) were sutured shut under anesthesia induced by $2-3 \%$ isofluorane in oxygen, otherwise according to the procedures described previously (Gordon and Stryker, 1996). Ages of animals at the beginning of MD were as follows: postnatal day 16 (P16)-P18 for precritical period animals, P24-P29 for critical period animals, and 12-13 weeks old for adult animals. Animals were checked daily for the first few days after surgery and occasionally there- 
after to make sure that the eyelids were completely sealed closed by the scar tissue. Mice whose eyelid fusion had a hole or those with any indications of corneal damage or cataract were removed from the study before imaging. Animals were maintained on a $12 \mathrm{~h}$ light/dark cycle with ad libitum access to food and water.

Optical imaging. Surgical preparation, visual stimuli, optical imaging of intrinsic signals, and data analysis were performed as described previously (Gordon and Stryker, 1996; Kalatsky and Stryker, 2003; Cang et al., 2005). Briefly, mice were anesthetized with an intraperitoneal injection of Nembutal $(50 \mathrm{mg} / \mathrm{kg})$ supplemented by chlorprothixene $(10 \mathrm{mg} / \mathrm{kg}$, i.m.), and placed in a stereotaxic frame. Atropine $(0.3 \mathrm{mg} / \mathrm{kg}$, s.c.) and dexamethazone $(2 \mathrm{mg} / \mathrm{kg}$, s.c.) were administered additionally to suppress secretions and brain edema, respectively. The animal's temperature was maintained at $37.5^{\circ} \mathrm{C}$ by a rectal thermoprobe feeding back to a heating pad. The heart rate was continuously monitored through electrocardiograph leads attached to the animal. This enabled us to keep the depth of anesthesia stable. A tracheotomy and a craniotomy were performed. The exposed left occipital cortex was covered with agarose and a coverslip to form an imaging window. The eyes were protected with a thin layer of silicon oil. For visual stimuli, a horizontal bar $\left(2^{\circ}\right.$ in height and $20^{\circ}$ in width) drifting up or down with a period of $8 \mathrm{~s}$ was presented for $320 \mathrm{~s}$ (i.e., 40 cycles) on a high-refresh-rate monitor positioned $25 \mathrm{~cm}$ in front of the animal. The axis of drifting was shifted $5^{\circ}$ rightward from the midline to elicit responses in the center of the binocular zone. Optical images of visual cortex were acquired continuously under a $610 \mathrm{~nm}$ illumination over the stimulation period with a Dalsa 1M30 CCD camera (Dalsa) equipped with a Nikon $135 \times 50 \mathrm{~mm}$ tandem lens. The Fourier component of light reflectance changes matched to the stimulus frequency was extracted pixel by pixel from the image stream to generate amplitude and phase maps of cortical intrinsic signals. In each imaging session, a set of four images was taken by visualizing the response of each eye (i.e., the contralateral or ipsilateral eye) to each direction (i.e., upward or downward). Then, a map with absolute retinotopy and average magnitude for each eye (called "average map") was computed from a pair of the upward and downward maps by stimulus reversal and averaging. The magnitudes of response in these maps are fractional changes in reflectance. For calculation of ocular dominance, the magnitude maps were smoothed by a uniform $5 \times 5$ filter and thresholded at $30 \%$ of peak response amplitude to define a response region. The extent of central binocular zone was defined by maps of the responses of the ipsilateral eye. The ocular dominance index (ODI) was calculated by averaging $(C-$ $I) /(C+I)$, where $C$ and $I$ represent the response magnitude of each pixel to the contralateral and ipsilateral eyes, respectively, over all responsive pixels in the region. The ODI ranges from +1 to -1 , where a positive value indicates a contralateral bias, and a negative value an ipsilateral bias. Maximum magnitude values are also presented as a measure of response strength separately for each eye. The ODIs and maximum magnitude values obtained independently from multiple sessions were averaged to determine representative values for each animal. For clarification, we expressed all magnitudes after multiplication by $10^{4}$ so that they can be written as small, positive numbers.

Imaging data from 102 mice are reported in Results. Measurements in four additional mice were not completed when initial responses to contralateral eye stimulation were $<0.9 \times 10^{-4}$, approximately one-third of normal, a sign of poor responsiveness. The data from these four mice were excluded from analysis.

$C P P$ injections. The competitive NMDA receptor antagonist $(R, S)-3$ (2-carboxypiperazin-4-yl)propyl-1-phosphonic acid (CPP) (Tocris Bioscience) was dissolved in saline at a concentration of $1-1.5 \mathrm{mg} / \mathrm{ml}$ and the drug solution was injected intraperitoneally at a dose of $10-15 \mathrm{mg} / \mathrm{kg}$ every $24 \mathrm{~h}$ (Villarreal et al., 2002). To animals with MD, the first CPP injection was made $4-6 \mathrm{~h}$ before MD surgery.

Tissue preparation and immunoblotting. Visual cortices were dissected using a blunted 16-gauge needle from 2-mm-thick occipital cortex slices cut on an acrylic matrix. Each tissue was separately homogenized by a 1.5 $\mathrm{ml}$ tube and a plastic pestle in $100 \mu \mathrm{l}$ of buffer containing $60 \mathrm{~mm}$ Tris$\mathrm{HCl}$, pH 6.8, 2\% SDS, 5 mm EDTA, and $1 \times$ Halt Proteinase Inhibitor Cocktail (Pierce). Homogenates were then incubated at $100 \mathrm{C}^{\circ}$ for $2 \mathrm{~min}$. Total protein concentrations were quantitated with the BCA Protein
Assay kit (Pierce). Protein samples ( $5 \mu \mathrm{g}$ protein/lane) were resolved by 7.5\% SDS-PAGE gels and transferred onto polyvinylidene difluoride membrane. Blots were incubated with rabbit polyclonal anti-Zif268 (otherwise called Egr-1) antibody (Santa Cruz Biotechnology) or mouse monoclonal anti- $\beta$ III tubulin antibody (Clone TUJ1; Millipore) followed by incubation with HRP-conjugated anti-rabbit or anti-mouse secondary antibody (Jackson ImmunoReseach Laboratories), respectively. Signal was detected on film with ECLplus reagents (GE Healthcare). Film images were then captured with a CCD camera and optical density of bands was measured with NIH Image J software. The relative amount of Zif268 protein was determined by in-blot comparison with diluted standards and was normalized to that of $\beta$ III tubulin from the corresponding lane.

Behavioral testing of visual acuity. Visual acuity of unrestrained, freely moving mice was measured with the virtual optomotor system (Prusky et al., 2004). Briefly, a mouse was placed onto an elevated platform in the center of a virtual-reality chamber, which comprises four 17 inch LCD monitors arranged into a square. Vertical sinusoidal gratings of $100 \%$ contrast were drawn on the screens at various spatial frequencies $(0.042-$ 0.514 cycles/degree) and were rotated at a speed of $12 \%$ by a Macintosh software (OptoMotry; CerebralMechanics). The mouse's behavior was observed for 12 min through a video camera positioned immediately above the platform. When the mouse faced the screen and moved its head smoothly in parallel with the rotation of the gratings, it was judged that the mouse tracked the gratings and could see them. To assess the acute behavioral effects of CPP injection, measurements were performed before and after the CPP administration in the same animals. At the end of each session, the highest spatial frequency that elicited noticeable tracking behavior was recorded as the threshold. Thresholds for the left and right eyes were determined separately with clockwise and counterclockwise rotation of the gratings, respectively. An average of those two values was taken as the representative threshold for each animal.

Statistics. All data represent mean \pm SEM. Statistical significance between two groups in imaging, biochemical and behavioral experiments was calculated by Mann-Whitney test, two-tailed unpaired $t$ test, and Wilcoxon's matched pairs test, respectively.

\section{Results}

\section{Effects of brief MD during and outside of critical period}

We used Fourier analysis-based intrinsic signal optical imaging to measure visual responses and plasticity in the binocular zone of mouse visual cortex (Kalatsky and Stryker, 2003; Cang et al., 2005). A $2 \times 20^{\circ}$ light bar was swept at $10 \mathrm{deg} / \mathrm{s}$ upward or downward through the binocular visual field at $8 \mathrm{~s}$ intervals, and images of reflected light at $610 \mathrm{~nm}$ were captured in a CCD camera at $7.5 \mathrm{~Hz}$ and $512 \times 512$ pixel by 16 bit resolution (Fig. $1 \mathrm{Aa}$ ). Responses of each pixel at the stimulus frequency were extracted by Fourier analysis. Data about magnitude and retinotopic position of the response at each pixel were obtained as amplitude and phase values of the Fourier components, respectively (Fig. 1Ab). For presentation, these two parameters are jointly depicted in a "polar map," where the response magnitude is expressed as brightness and the retinotopy as hue (Fig. 1D). The ODI was calculated by averaging the ratios of response magnitudes to the two eyes across the response area (see Materials and Methods). This technique provides a quantitative, unbiased measurement of response magnitude. The relative responses to the two eyes measured by optical imaging are closely correlated with similar measures made by single-unit recording (Gordon and Stryker, 1996; Cang et al., 2005).

We first compared the effects of brief 4-5 d MD on the responsiveness of the visual cortex to the two eyes when the deprivation was performed at the peak of the critical period with the effects of the same period of deprivation in younger and older animals. In nondeprived control mice of all ages, the magnitude of the contralateral-eye response was $\sim 1.5-1.7$ times larger than 
that of the ipsilateral eye. This gave rise to an average $\mathrm{OD}$ index of $\sim 0.22$ (Fig. $1 B$ ). In the precritical period ( $\mathrm{MD}$ starting at P16-P18), mice subjected to 4-5 d MD showed only slight plasticity, with an OD barely lower than the nondeprived control mice $(0.22 \pm 0.026$ vs $0.16 \pm 0.021 ; p=$ 0.20 ) (Fig. $1 \mathrm{Ba}$ ). This minor reduction in OD index was mediated by a small decrease in the response of the deprived eye $(2.80 \pm 0.20$ vs $2.31 \pm 0.03 ; p=0.10)$ (Fig. $1 C a, D a)$. In contrast, at the peak of the critical period (MD starting at P24-P29) mice showed great plasticity after brief $M D$, with a significantly lower OD index than the nondeprived control group $(0.22 \pm 0.016$ vs $0.02 \pm 0.025 ; p<0.01)$ (Fig. $1 \mathrm{Bb}$ ). The degree of this OD shift was comparable with the OD shift observed in single-unit recordings (Gordon and Stryker, 1996). This plasticity was principally the result of a dramatic decrease of the deprived-eye response $(2.96 \pm 0.27$ vs $2.07 \pm 0.09 ; p<0.05$ ) (Fig. $1 C b, D b)$. At 12 weeks of age, when mice are considered to be adult, brief MD produced a much weaker OD shift than during the critical period $(0.26 \pm 0.022$ vs $0.18 \pm 0.039 ; p=$ 0.10 ) (Fig. $1 B c$ ). This small shift in OD index resulted from a small decrease of the deprived eye response $(2.68 \pm 0.34$ vs $2.02 \pm 0.26 ; p=0.15$ ) (Fig. $1 C c, D c$ ). Together, these results suggest that brief MD causes a substantial decrease in deprivedeye responsiveness only at the peak of the critical period, with much smaller reductions before and after.

Time courses of OD shifts after MD in juvenile and adult mice

We next examined the effects of varying durations of $\mathrm{MD}$ on cortical responses to the two eyes in adult mice and in juvenile mice during the critical period. In juvenile mice, a very short MD for 1-2 d elicited a significant, intermediate shift in OD $(0.12 \pm 0.022)$ (Fig. $2 A)$. A brief MD for 4-5 d gave a near-saturating OD shift, with a mean ODI of $0.02 \pm 0.025$ (Figs. $1 B b, 2 A)$. As with single-unit recording (Gordon and Stryker, 1996), longer MD caused only modest additional change in OD toward the slightly negative value observed at 13-22 d after MD (-0.04 \pm 0.026 ) (Fig. 2A). The OD shift in the critical period was the result of an initial decrease in the deprived-eye response followed by a smaller increase in the response of the nondeprived eye (Fig. $2 B$ ) (deprived eye: no MD, $2.96 \pm 0.27$ vs $4-22 \mathrm{~d} \mathrm{MD}$, $2.15 \pm 0.09, p<0.05$; nondeprived eye: 0-5 d MD, $1.85 \pm 0.11$ vs 7-22 d MD, $2.22 \pm 0.13, p<0.05)$. Overall, these re-
A a

$\mathrm{b}$

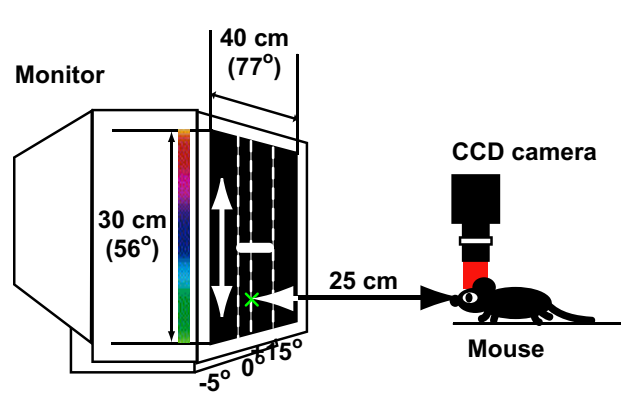

B

a Pre-CP

b CP
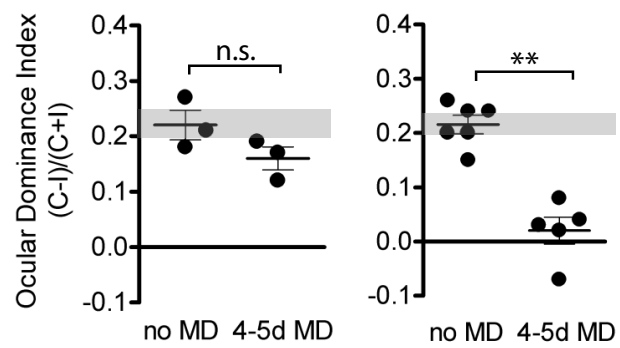

a Pre-CP

C

b CP

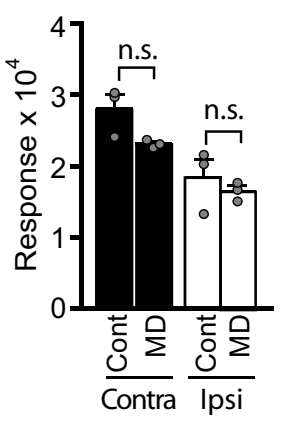

a

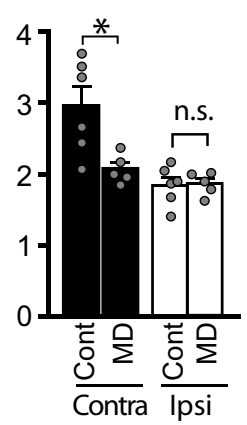

b

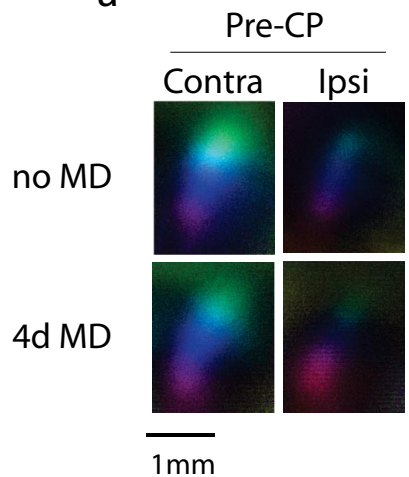

CP
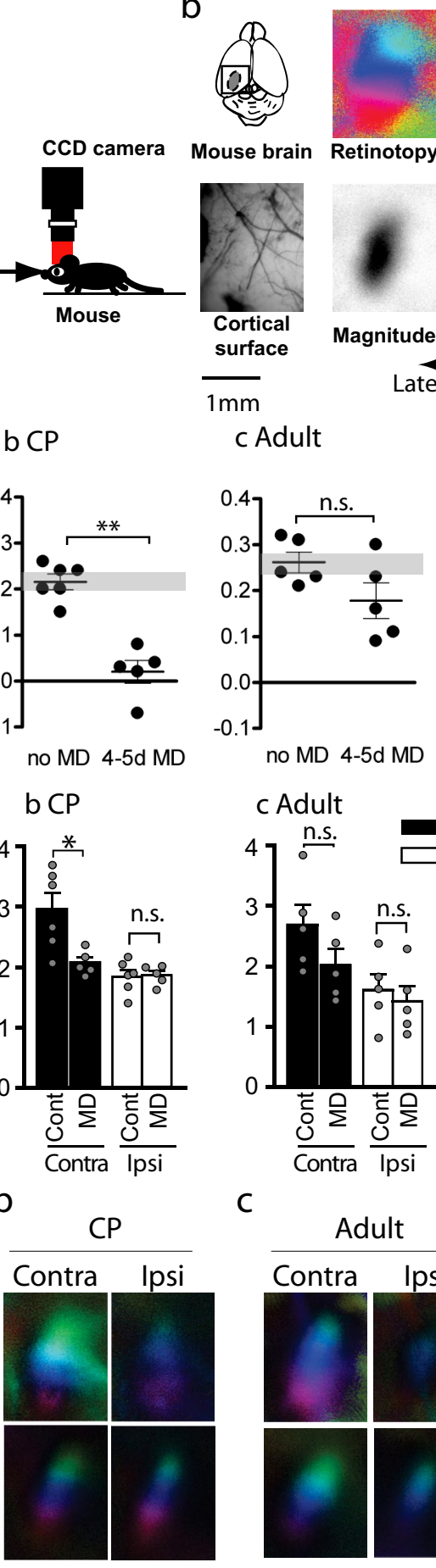

Retinotopy

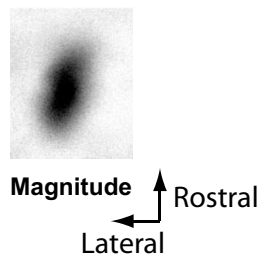

$1 \mathrm{~mm}$

c Adult
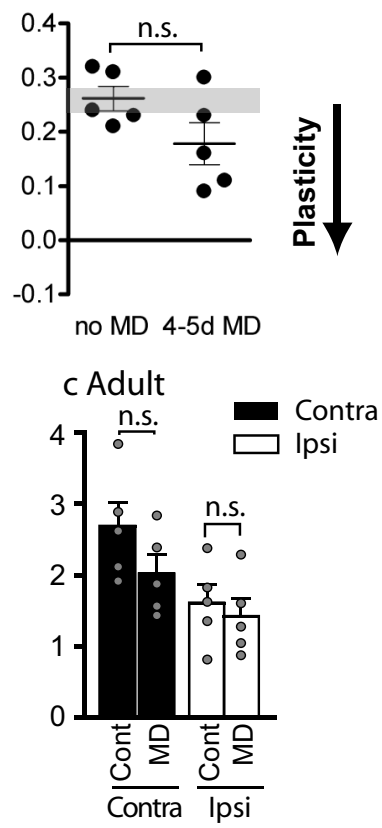

C

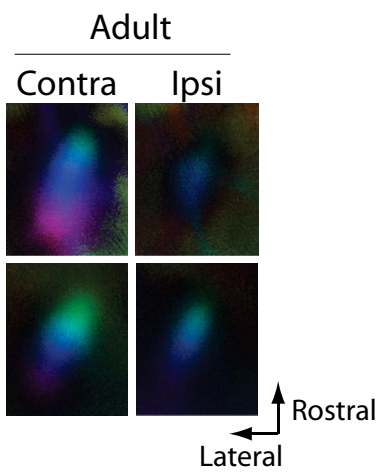

Figure 1. The effects of brief MD on mice of different ages. Aa, A short sweeping bar moving upward or downward was presented in the binocular visual field of a mouse and the evoked cortical activity was imaged with a CCD camera. Ab, 0ccipital cortex of left hemisphere was exposed (top left) and cortical blood vessel pattern was captured under green light (bottom left). Activity maps of retinotopy (top right) and magnitude (bottom right) were imaged under red light to create polar maps as seen hereafter. $\boldsymbol{B} \boldsymbol{a}-\boldsymbol{B C}, 0 D$ index after brief (4-5d) MD of the contralateral eye in precritical period mice ( $\boldsymbol{B a}$, Pre-CP; $n=3$ each), critical period mice ( $B \boldsymbol{b},(\boldsymbol{C} ; n=5-6)$ or adult mice ( $\boldsymbol{B} \boldsymbol{C}$, Adult; $n=5$ each). The $y$-axis shows OD index as described in Materials and Methods. The mice subjected to brief MD (MD) showed a significantly lower $0 D$ index in $C P$, but neither in pre- $C P$ nor adulthood. The downward arrow shown to right illustrates that a lower OD index after MD indicates greater plasticity. ${ }^{* *} p<0.01$. n.s., Not significant. Ca-Cc, Maximum response magnitude expressed as fractional change in light reflectance elicited by stimulation of the contralateral (black bars) or ipsilateral (white bars) eye of mice deprived before ( $\mathbf{C} \boldsymbol{a})$ or during $(\boldsymbol{C} \boldsymbol{b})$ the critical period or in adulthood $(\mathbf{C C})$. Brief MD reduced responsiveness of the deprived eye significantly only in $C P$, but not pre-CP or in adulthood. ${ }^{*} p<0.05$. Da-Dc, Polar maps of responses in binocular visual cortex of mice of different ages with or without brief MD. 
A

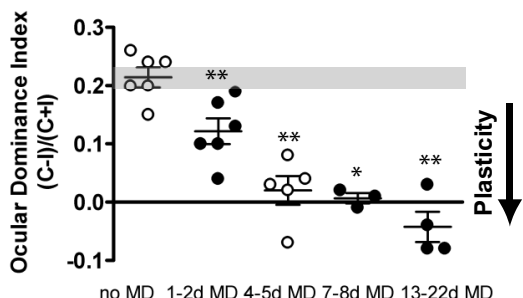

B

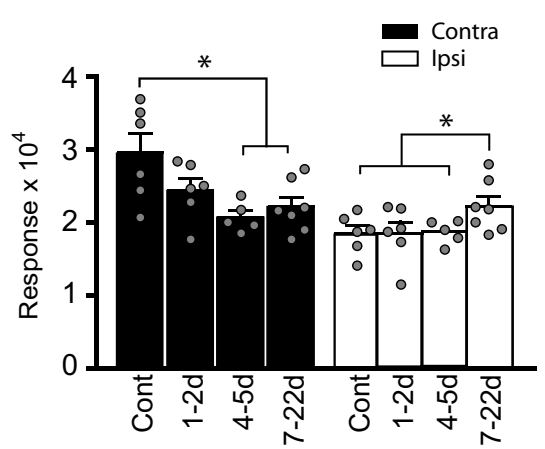

$\mathrm{E}$

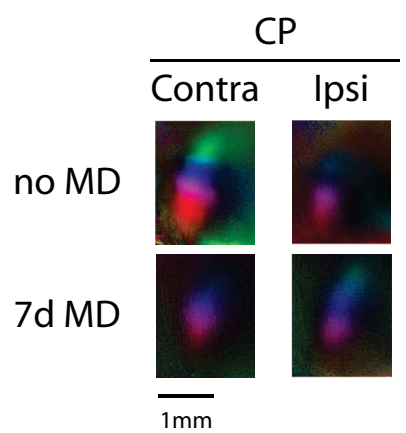

C

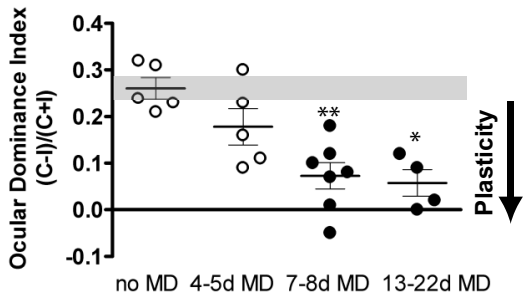

D
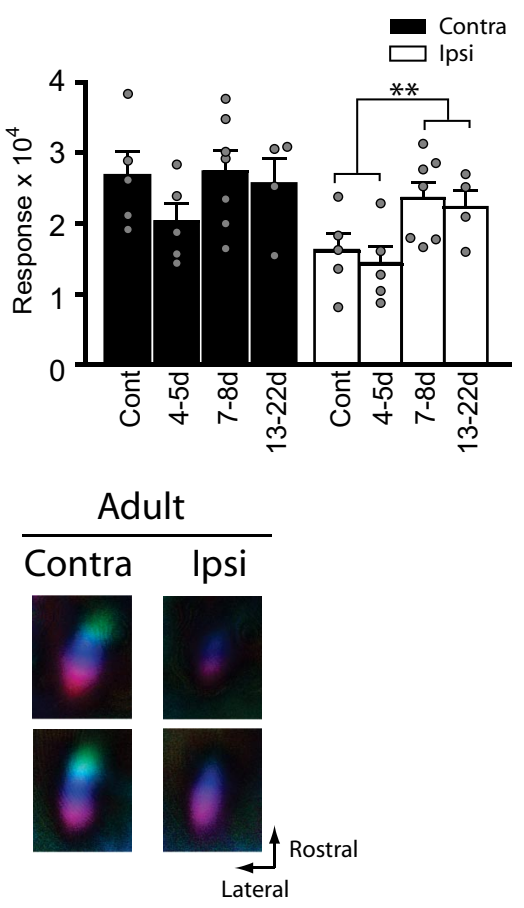

Figure 2. Critical period ocular dominance plasticity and adult ocular dominance plasticity show different time courses and response changes. $A$, Time course of $0 D$ shifts in the critical period after varying lengths of $M D$. Data indicated by open circles were taken from Figure 1 for comparison $\left(n=3-6 ;{ }^{*} p<0.05,{ }^{* *} p<0.01\right)$. B, Maximum magnitude of responses elicited by the stimulus to the contralateral (Contra; black bars) or ipsilateral (Ipsi; white bars) after varying length of MD in critical period mice. For statistical comparison, data from groups that were qualitatively indistinguishable were pooled as indicated ( $\left.n=6-17 ;{ }^{*} p<0.05\right)$. Cont, Control. $C$, Time course of $O D$ shift in adult mice after varying length of $M D$. Data indicated in open circles were taken from Figure 1 for comparison $\left(n=4-7 ;{ }^{*} p<0.05,{ }^{* *} p<0.01\right)$. D, Maximum magnitude of responses elicited by the stimulus to the contralateral (black bars) or ipsilateral eye (white bars) in adult mice. Data from groups that did not show qualitative difference were pooled as indicated for statistical comparison $(n=10-11 ; * * 0.01) . E$, Polar maps of cortical responses in critical period and adult mice with or without $7 \mathrm{~d}$ MD.

sults are consistent with earlier studies in cats (Mioche and Singer, 1989) and mice (Frenkel and Bear, 2004), and demonstrate that the OD shift during the critical period is rapid, large, and involves an initial sharp decrease of the deprived-eye response (Fig. 2E).

In contrast, in adult mice, $4-5 \mathrm{~d}$ of $\mathrm{MD}$ was not effective in inducing a significant OD shift (Figs. $1 B c, 2 C$ ). However, $7 \mathrm{~d} \mathrm{MD}$ did result in a significant OD shift $(0.07 \pm 0.028 ; p<0.01$ vs control) (Fig. 2C). Longer MD, for 13-22 d, gave rise to an OD index similar to that caused by $7-8 \mathrm{~d} \mathrm{MD}(0.06 \pm 0.028 ; p<0.05$ vs control). This indicates that $7-8 \mathrm{~d} \mathrm{MD}$ has a saturating effect on the OD of adult visual cortical responses and that the saturating MD in adulthood does not produce as great a change as was seen in the critical period. Thus, OD plasticity in adulthood (aODP) is slower in time course and milder in degree than the OD shift in the critical period.

An analysis of changes in response magnitude for each eye showed that aODP was initiated by a small decrease in the responsiveness of the deprived eye at 4-5 d after MD (Figs. 1Cc, 2D). To our surprise, however, the response magnitude of the deprived eye at $7 \mathrm{~d}$ after MD appeared to recover from this weakening, returning to a level comparable with that of nondeprived control mice $(2.74 \pm 0.29)$. The magnitude of the nondeprived eye response also increased markedly after deprivations longer than $7 \mathrm{~d}(2.32 \pm$ $0.16 ; p<0.01,0-5 \mathrm{~d}$ MD vs $7-22 \mathrm{~d}$ MD) (Fig. $2 D, E$ ). The response magnitudes for both eyes at 13-22 d after MD were qualitatively indistinguishable from those after 7-8 d MD (2.56 士 0.36 , contralateral; $2.23 \pm 0.25$, ipsilateral), confirming that a week of MD is sufficient to induce a full expression of aODP. The delayed increases in responses to both eyes are much more substantial in aODP than in the critical period form, and the reduction in response to the deprived eye is much less so. Collectively, these results suggest that aODP is not merely a slower form of the OD plasticity in the critical period but rather is mediated by a different series of response changes, one in which strengthening plays a key role.

\section{Effects of ipsilateral MD and BD}

OD plasticity in the critical period has been shown to be competitive, with complementary changes in the responses to the two eyes and little effect of binocular deprivation (BD) (Gordon and Stryker, 1996). To investigate further how visual activity from the two eyes interacts in aODP, we next examined the effects of the ipsilateral-eye $\mathrm{MD}$ and BD in adult mice and in juvenile mice during the critical period.

In juvenile mice, a brief ipsilateraleye MD for 4-5 d produced substantial plasticity, of a similar magnitude but opposite in direction to the plasticity produced by deprivation of the other eye $(0.38 \pm 0.012$, experimental vs $0.22 \pm 0.016$, control; $p<0.05$ ) (Fig. $3 A$ ). This finding suggests that capacity for OD plasticity is symmetrical between the two hemispheres during the critical period. An examination of response magnitude revealed that plasticity after ipsilateral-eye MD was mediated primarily by a decrease in the responsiveness of the deprived eye (from $1.85 \pm 0.11$ to $1.26 \pm$ $0.17 ; p<0.05$ ) (Fig. $3 B, C$ ). This observation matches the decrease in the responsiveness of the contralateral eye after contralateral-eye MD and thus indicates that a rapid loss of responsiveness to the deprived eye is a common mechanism for MD-induced OD shifts in the critical period.

Confirming previous single-unit findings (Gordon and Stryker, 1996), a brief period of BD in juvenile mice for $4-5 \mathrm{~d}$ produced no change in $\mathrm{OD}(0.20 \pm 0.015 ; p=0.38)$ (Fig. $3 A)$. Response magnitudes for both eyes after BD did not differ signif- 
icantly from those of nondeprived control mice $(2.72 \pm 0.12$ for the contralateral eye, $p=0.90$ vs control, $1.91 \pm 0.16, p=0.71)$ (Fig. $3 B, C$ ). Together, results of ipsilateral-eye $\mathrm{MD}$ and $\mathrm{BD}$ in juvenile mice reveal a capacity for plasticity for input from the ipsilateral eye similar to that for the contralateral eye input during the critical period. This symmetry is reflected in the lack of an OD shift when both eyes are deprived.

In adult mice, the effects of deprivation on the contralateral and ipsilateral pathways are no longer symmetrical. Ipsilateral-eye MD for 4-15 d produced no significant change in OD and only the slightest change in the average values (control, $0.21 \pm 0.020 ; 4-5 \mathrm{~d}$ ipsilateral MD, $0.24 \pm 0.038, p=0.39$ vs control; $7-15 \mathrm{~d}$ ipsilateral MD, $0.26 \pm 0.027, p=0.19$ vs control) (Fig. 3D). The degree of the OD shift after 7-15 d ipsilateral-eye MD was much smaller $(36 \%)$ than that after the contralateral MD. The response magnitude of the deprived ipsilateral eye also did not change significantly (Fig. $3 E, F$ ). Response magnitude of the nondeprived contralateral eye after 7-15 d ipsilateral MD also changed little, increasing slightly but not significantly (control, $3.05 \pm 0.21$; $7-15 \mathrm{~d}$ ipsilateral MD, $3.34 \pm 0.25, p=$ 0.56 vs control).

The striking asymmetry in the adult between ipsilateral- and contralateral-eye deprivation raises the possibility that adult plasticity depends on the relative strength of the input being deprived. The effects of $\mathrm{BD}$ in the adult give additional support to this property of adult plasticity. Adult mice subjected to $7 \mathrm{~d}$ BD exhibited a significant shift in OD (from $0.21 \pm 0.020$ to $0.12 \pm 0.021 ; p<0.05$ ) (Fig. 3D). The surprising $\mathrm{OD}$ shift induced by $\mathrm{BD}$ had the same direction of change as the OD shift after the contralateral-eye MD but was milder (69\%) in degree. Response magnitudes for both eyes after $7 \mathrm{~d}$ BD appeared larger than those of nondeprived controls, significantly so for the ipsilateral eye (from $2.19 \pm 0.12$ to $3.23 \pm$ $0.24 ; p<0.05)$ although not for the contaralateral eye $(3.74 \pm$ $0.30 ; p=0.19$ ) (Fig. $3 E, F)$. The result of both ipsilateral-eye MD and $\mathrm{BD}$ suggest that plasticity in the adult visual cortex is simply not much affected by the presence or absence of visual stimulation through the ipsilateral eye. When the ipsilateral eye is deprived alone, there is no significant plasticity, and when it is deprived together with the contralateral eye, the effects are similar to those of depriving the contralateral eye alone. Together, these results suggest that aODP obeys its own plasticity rules, different from those that operate during the critical period.

\section{Role of NMDA receptor activation}

Finally, to gain insights into possible difference in the molecular mechanisms that underlie critical period and adult plasticity, we ipsilateral.
D

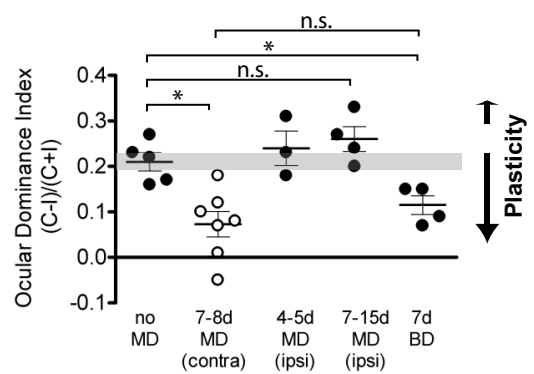

$\mathrm{E}$

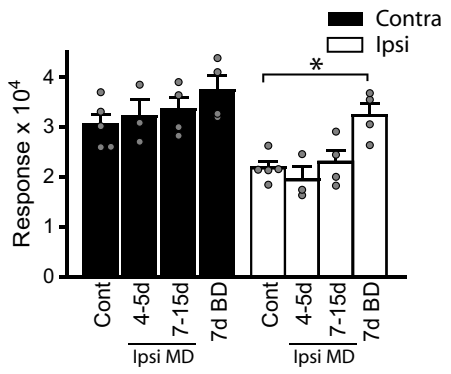

$\mathrm{F}$

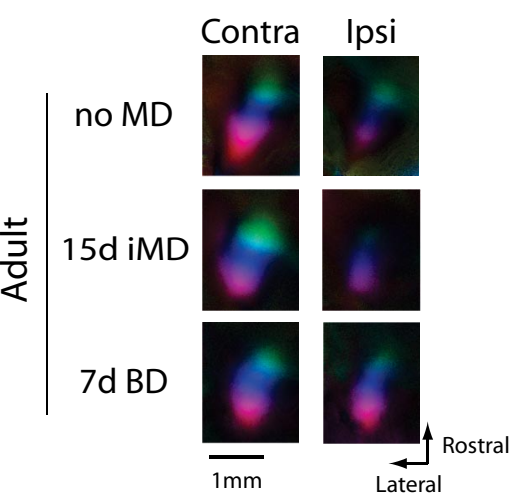

Figure 3. Effects of ipsilateral MD and BD in juvenile and adult mice. $A, O D$ shifts after 4 d ipsilateral-eye $M D$ and BD in critical period mice. The $0 D$ bias shifted toward the contralateral nondeprived eye after 4 d ipsilateral MD. BD did not change OD $(n=$ magnitude for each eye after $4 \mathrm{~d}$ ipsilateral-eye MD and BD. The value for the ipsilateral closed eye response of mice with $4 \mathrm{~d}$ ipsilateral MD was significantly smaller than that of control mice ( ${ }^{*} p<0.05$, Cont Ipsi vs Ipsi MD Ipsi; $\left.n=3-6\right)$. C, Polar maps of mice. OD changed only very slightly toward the contralateral nondeprived eye after ipsilateral-eye MD. BD caused 作 response magnitude of the ipsilateral eye after $\mathrm{BD}$ is significantly larger than that of mice without deprivation $\left(n=4-5\right.$; ${ }^{*} p<$ 0.05). F, Polar maps of cortical responses in adult mice with no MD, $7 \mathrm{~d}$ ipsilateral MD, and BD. Contra, Contralateral; Ipsi,

investigated the role of NMDA-receptor activation in the OD shifts of juvenile and adult mice. We selectively blocked NMDA receptor activity by repeated intraperitoneal injection of 10-15 $\mathrm{mg} / \mathrm{kg}$ of the competitive antagonist CPP during a period of MD. This drug has been shown to cross the blood-brain barrier to the brain after systemic administration and to inhibit the NMDA receptor activity effectively as long as $24 \mathrm{~h}$ after injection in rodents (Villarreal et al., 2002; Frenkel et al., 2006). Indeed, in control juvenile and adult mice, we confirmed that intraperitoneally administered CPP significantly suppressed NMDA receptor-dependent expression of the immediate early gene product Zif268 within visual cortex at $24 \mathrm{~h}$ after injection (Mataga et al., 2001) (Fig. 4A). This biochemical finding allowed us to schedule daily CPP injections for continuous inhibition of NMDA receptor activity in visual cortex. Behavioral observation 
A

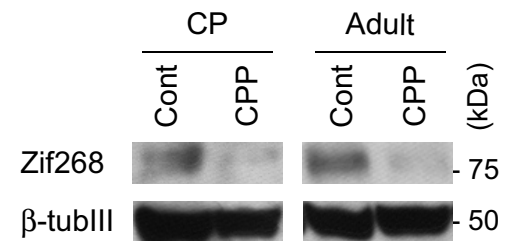

B

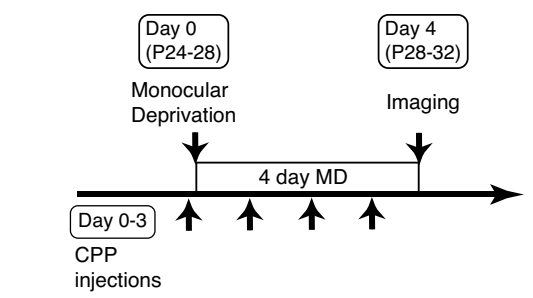

D

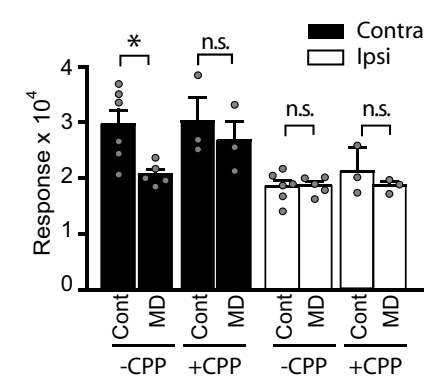

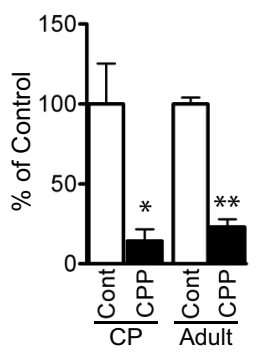

C

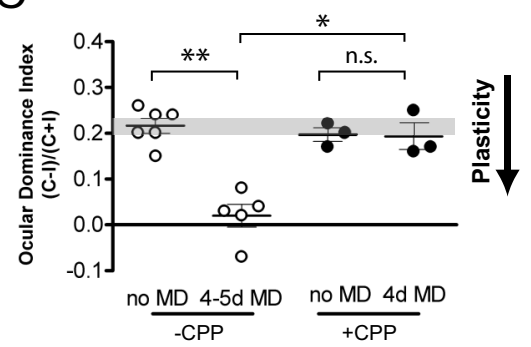

E

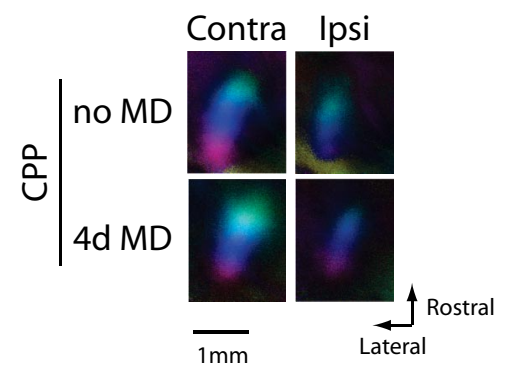

Figure 4. CPP-mediated suppression of NMDA receptor function in visual cortex and NMDA receptor-dependent ocular dominance plasticity in the critical period. $A$, Suppression of Zif268 expression in visual cortex at $24 \mathrm{~h}$ after CPP injection in juvenile and adult mice. (PP or control saline (Cont) were injected into mice in the critical period at P28 and in adulthood at P80 and the amount of zif268 (top) and $\beta$ III tubulin (bottom) proteins at $24 \mathrm{~h}$ after injection was quantified by immunoblotting. The right panel shows summary data $\left(n=3\right.$ each; $\left.{ }^{*} p<0.05,{ }^{* *} p<0.01\right)$. B , Mice in the critical period were treated with CPP $4-6 \mathrm{~h}$ before MD on day 0 and every $24 \mathrm{~h}$ thereafter and were imaged at $4 \mathrm{~d}$ after MD. C, OD shifts after $4 \mathrm{~d} M D$ were blocked by daily CPP treatment in critical period mice $\left(n=3-6 ;{ }^{*} p<0.05,{ }^{* *} p<0.01\right)$. Data indicated in open circles were taken from Figure 1 for comparison. $D$, Decrease in the response of the deprived eye after $4 \mathrm{~d} M D$ was prevented by daily CPP treatment in critical period mice $(n=$ 3-6).E, Polar maps of cortical responses of critical period mice with or without CPP treatment and MD. Contra, Contralateral; Ipsi, ipsilateral.

during the first 30-60 min after injection confirmed that this dose of CPP did not cause any obvious behavioral abnormality such as hyperlocomotion, stereotypy, or lethargy, and casual observation did not distinguish between experimental and control animals. Quantitative assessment of visually evoked optokinetic behavior further supported these observations: visual acuity thresholds did not change 30-90 min after CPP injection in juvenile mice (before CPP, $0.340 \pm 0.014$ cycles/degree vs $0.5 \mathrm{~h}$ after CPP, $0.349 \pm 0.012$ cycles/degree; $p=0.68$ ), and only minimal suppression was recognizable at $30-90 \mathrm{~min}$ in adult mice (before CPP, $0.378 \pm 0.004$ cycles/degree vs $0.5 \mathrm{~h}$ after CPP, $0.352 \pm 0.004$ cycles/degree; $p<0.05)$ but not at $3-6 \mathrm{~h}$ after injection ( $3 \mathrm{~h}$ after CPP, $0.374 \pm 0.004$ cycles/degree; $p=0.70$ vs before CPP) (supplemental Fig. 1, available at www.jneurosci.org as supplemental material).

We first examined the effect of NMDA receptor inhibition on the OD shift after brief MD during the critical period. CPP (10 $\mathrm{mg} / \mathrm{kg}$ ) was first injected $4-6 \mathrm{~h}$ before the start of MD and was repeatedly administered every $24 \mathrm{~h}$ until the day before imaging (Fig. $4 B$ ). The OD shift after $4-5 \mathrm{~d} \mathrm{MD}$ in juvenile mice was completely blocked by the CPP treatment (Fig. 4C). The OD index of CPP-treated deprived mice remained within the range of that of the nondeprived control and was significantly higher than that of control deprived mice $(0.19 \pm 0.028, p=0.70$ vs nondeprived control; $p<0.05$ vs deprived control). CPP treatment by itself, with no $\mathrm{MD}$, had no effect on OD (Fig. $4 C$ ) or on response magnitude (Fig. 4D,E). Measurements of response magnitudes after $\mathrm{MD}$ showed that $\mathrm{CPP}$ treatment prevented the loss of responsiveness to the deprived eye (Fig. $4 D, E$ ). These findings suggest that the blockade of plasticity by CPP treatment was mediated by its selective action on NMDA receptors rather than by a more general inhibition of neural activity. They are in agreement with previous single-unit findings in cats that OD plasticity in the critical period is NMDAreceptor dependent (Kleinschmidt et al., 1987).

In adult mice, as in juvenile mice, the effectiveness of CPP on blocking NMDA receptors in visual cortex was confirmed by marked reduction of Zif268 expression at $24 \mathrm{~h}$ after injection (Fig. $4 \mathrm{~A}$ ). Plasticity was almost completely blocked by CPP treatment throughout the $7 \mathrm{~d}$ period of MD necessary to reveal plasticity in control adult animals (ODI, $0.21 \pm 0.022, p=$ 0.90 vs control, $p<0.05$ vs $7 \mathrm{~d}$ MD) (Fig. 5A,B). To determine whether the response-strengthening phase of aODP is separable from its earlier phase characterized by the reduction in deprived-eye response, we blocked NMDA receptor activation in another group of adult animals only during the last $3 \mathrm{~d}$ of the $7 \mathrm{~d}$ period of MD (Fig. 5A). Consistent with this hypothesis, these mice showed at most a very small OD shift, comparable with untreated animals after $4 \mathrm{~d}$ of MD (Fig. $5 B$ ). As expected, CPP treatment during a $4 \mathrm{~d} \mathrm{MD}$ in adult animals had no significant effect (supplemental Fig. 2, available at www.jneurosci.org as supplemental material). Notably, the CPP treatment did not impair visual responses (Fig. 5C). These results support the hypothesis that aODP, like critical period plasticity, requires NMDA receptor activation.

\section{Discussion}

In this study, we have made a detailed comparison of the effects of visual deprivation on binocular cortical responses in adult and juvenile mice using the Fourier optical imaging technique. The results are summarized in Fig. 6. In visual cortex of juvenile mice, $4 \mathrm{~d} \mathrm{MD}$ is sufficient to elicit an NMDA receptor-dependent, nearsaturating OD shift (Fig. 6A). This rapid shift is mediated primarily by a large reduction of the response of the deprived eye (Fig. 6A). Ipsilateral MD produced an OD shift that is comparable in magnitude but opposite in sign to that caused by contralateral MD. BD has no effect on responses to the two eyes (Fig. $6 B$ ). In contrast, $7 \mathrm{~d} \mathrm{MD}$ of the contralateral eye is required to produce 
a significant and saturating OD shift in adult visual cortex. This shift was initiated by a small and transient decrease in the deprived eye's response at $4 \mathrm{~d}$ after MD, and was followed by an increase of the response of the nondeprived eye at $7 \mathrm{~d}$ after $\mathrm{MD}$ and a recovery of the response of the deprived eye from its initial decrease (Fig. 6C). This plasticity process in adulthood is also dependent on NMDA receptor activation. MD of the weaker ipsilateral eye is very much less effective in eliciting an OD shift than that of the contralateral eye. BD caused a modest OD shift similar to the effect of MD of the contralateral eye alone (Fig. 6D). These findings reveal that aODP differs qualitatively from that in the critical period in several respects.

\section{Comparison with previous studies}

Experience-dependent OD plasticity in adult rodent visual cortex has been studied with evoked potentials (Sawtell et al., 2003; Pham et al., 2004; Sale et al., 2007), singleunit recording (Antonini et al., 1999; Fischer et al., 2007), measurements of visual acuity (Prusky et al., 2006), immediate early gene expression (Tagawa et al., 2005), and intrinsic signal optical imaging (Hofer et al., 2006b). The present study adds two novel findings to the previous literature and confirms others. (1) We have shown that aODP consists of two phases that are even more distinct than those in juvenile animals: an initial reduction of contralateral deprived-eye response, followed by a second phase in which responses to both eyes increase considerably. The mechanism of adult OD plasticity was first described as being mediated almost exclusively by potentiation of the nondeprived response (Sawtell et al., 2003; Hofer et al., 2006b), although this view has been expanded to include both depression of the deprived eye response and potentiation of the open-eye response (Frenkel et al., 2006). The potent recovery of the deprived eye response from its transient depression that we find here is novel and shows some similarity to the potentiation of the response to the deprived vibrissa after long-term whisker deprivation in rat barrel cortex (Glazewski and Fox, 1996). (2) We find here that BD in adult mice, unlike in juveniles, induces a substantial OD shift. This novel observation is a qualitative difference between plasticity during critical period and adulthood. (3) We also find in adult but not juvenile animals a clear asymmetry between the two hemispheres in the effects of deprivation in the adult, which may be related to the effects of $\mathrm{BD}$. This asymmetry is a remarkable feature of aODP. For both BD and ipsilateral-eye deprivation, it appears that reducing the activity of the less-effective ip-
A

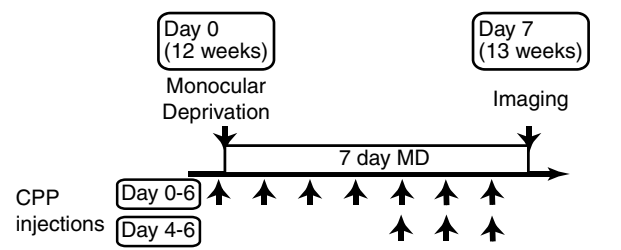

C

\section{B}

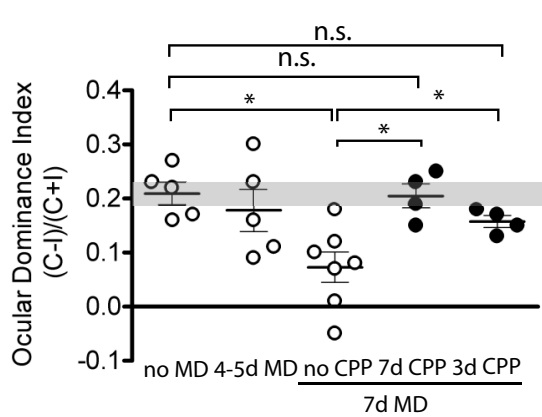

Figure 5. Role of NMDA receptor activation in adult ocular dominance plasticity. $A, \ln 7 \mathrm{~d}$ (PP treatment (days $0-6$ ), adult mice were treated with $(P P 4-6 \mathrm{~h}$ before MD on day 0 and every $24 \mathrm{~h}$ thereafter, and were imaged at $7 \mathrm{~d}$ after $\mathrm{MD}$. $\ln 3 \mathrm{~d}$ CPP treatment (days 4 - 6), adult mice were subjected to $7 \mathrm{~d} \mathrm{MD}$ with no CPP treatment for the first $4 \mathrm{~d}$. Daily CPP injection was then started from day 4 to day $6 . B, 0 D$ shifts after $7 \mathrm{~d} M D$ in adult mice were fully blocked by $7 \mathrm{~d}$ CPP treatment $\left(n=4-7 ;{ }^{*} p<0.05\right)$. Mice with $3 \mathrm{~d}$ CPP treatment showed partial blockade of OD shifts compared with the full effect produced by $7 \mathrm{~d} M \mathrm{MD}\left(n=4-7 ;{ }^{*} p<0.05\right)$. Data indicated in open circles were taken from Figures 1 and 2 for comparison. C, Polar maps of cortical responses in adult mice with or without CPP treatment and MD. Contra, Contralateral; Ipsi, ipsilateral.
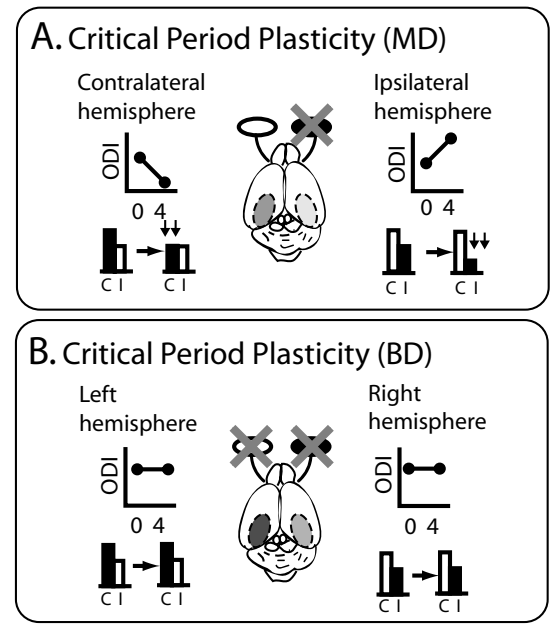

Figure 6. Different functional consequences for binocular responsiveness in the critical period and adult visual cortices after monocular and binocular deprivation. $\boldsymbol{A}$, In juvenile mice, brief $(4 \mathrm{~d})$ MD causes robust shift in ocular dominance toward the open eye in both the contralateral and ipsilateral hemispheres. These processes are mediated primarily by rapid decreases in the responsiveness of the deprived eyes. The degree of plasticity is equal in the two hemispheres. $\boldsymbol{B}$, BD has no effect on ocular dominance, suggesting that OD shifts produced by brief MD in the critical period are based on competition between inputs from the two eyes. C, In contrast, in adult mice, longer (7 d) MD causes a significantly large shift in the contralateral hemisphere to the deprived eye and only a hint of OD shift in the ipsilateral hemisphere. These shifts are mediated by transient small decreases in the response of the deprived eye at $4 \mathrm{~d}$ after MD followed by recovery from the depression of the response of the deprived eye and an increase in the response to the nondeprived eye at $7 \mathrm{~d}$ after MD. This asymmetry suggests that the degree of plasticity is determined by the strength of the deprived input. $D, B D(7 \mathrm{~d})$ causes a mild and atypical OD shift. This implies that the OD shifts in adult visual cortex are not based purely on competition in the conventional sense.

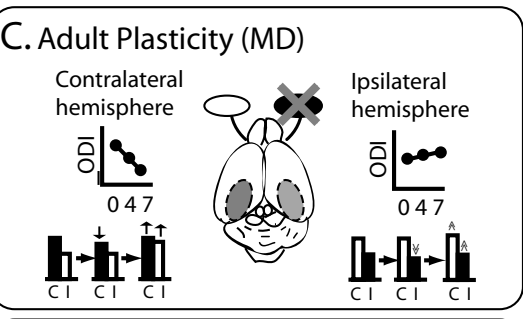

D. Adult Plasticity (BD)

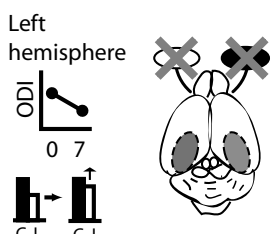

Right hemisphere 힌

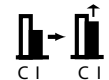


Table 1. A comparison of characteristics of ocular dominance plasticity in the critical period and adulthood

\begin{tabular}{|c|c|c|}
\hline & Critical period & Adult \\
\hline Time course & $\begin{array}{l}4 \mathrm{~d} \text {, primarily a rapid loss of re- } \\
\text { sponses of the deprived eye }\end{array}$ & $\begin{array}{l}7 \mathrm{~d} \text {, transient and slight loss of } \\
\text { responsiveness of deprived } \\
\text { eye, with prominent de- } \\
\text { layed potentiation of re- } \\
\text { sponses of both eyes }\end{array}$ \\
\hline Symmetry & $\begin{array}{l}\text { Symmetric, similar effects of con- } \\
\text { tralateral-eye and ipsilateral-eye } \\
\text { MD } \\
\text { No effect of BD }\end{array}$ & $\begin{array}{l}\text { Asymmetric, much larger } \\
\text { effect for contralateral-eye } \\
\text { MD } \\
\text { Clear effect of } B D \text {, like that of } \\
\text { contralateral-eye MD }\end{array}$ \\
\hline Involvement of NMDA receptors & Yes & Yes \\
\hline Trigger for plasticity & Imbalance of inputs & $\begin{array}{l}\text { Changes in cortical activity } \\
\text { levels }\end{array}$ \\
\hline Possible role of plasticity & Developmental rewiring & $\begin{array}{l}\text { Compensatory to maintain } \\
\text { activity }\end{array}$ \\
\hline
\end{tabular}

ticity that was observed in that case. (4) We have shown that aODP can be observed under barbiturate anesthesia. Previous findings suggested that adult OD plasticity may have been overlooked because barbiturate anesthetics whose actions augment cortical GABAergic inhibition might mask it (for review, see Hofer et al., 2006a; Karmarkar and Dan, 2006). Indeed, acute pentobarbital administration masked the effect of adult MD in VEP recordings (Pham et al., 2004). Our use of chlorprothixene in combination with barbiturate anesthesia apparently averts this issue.

Together with the previous studies, our new results on adult OD plasticity suggest that the visual cortex does not abruptly lose its capacity for plasticity at the end of the critical period, but rather that the characteristics of plasticity change over the course of maturation. One would expect that the precise neural connections that are altered as a result of $\mathrm{MD}$ and $\mathrm{BD}$ are also different at different ages. Future research will be needed to reveal which changes in connections within the visual cortex are responsible for the different changes in neuronal responses observed in the critical period and adulthood.

\section{Distinctive features of aODP}

We summarize basic features of juvenile and adult plasticity in Table 1. Critical period plasticity is primarily mediated by NMDA-receptor-dependent, rapid and substantial weakening of the deprived-eye response within $4 \mathrm{~d}$. It is triggered by imbalanced visual inputs provided by MD of either eye, and its capacity is symmetrical in the two hemispheres regardless of the different strengths of the ipsilateral- and contralateral-eye inputs. These features are consistent with a role for juvenile plasticity in the developmental refinement of cortical circuitry for binocular vision. However, aODP is a slower process, more modest in degree, that begins with a transient weakening of the deprived-eye response and is followed by its subsequent recovery and the strengthening of the open-eye response over $7 \mathrm{~d}$. Although it shares a dependence on NMDA receptor activation with critical period plasticity, it differs in that the magnitude of aODP depends strongly on the strength of the input being deprived, which results in asymmetry of plasticity between the two hemispheres. It is also triggered by changes in absolute activity level brought about by BD. Therefore, aODP appears primarily to be a compensatory process for maintaining activity levels in the adult visual cortex.

The synaptic mechanisms that underlie the distinctive prop- erties of aODP are not yet known. There are, however, age-dependent changes in the mechanisms of NMDA-receptor dependent long-term potentiation in rodent cortex (Yoshimura et al., 2003). Homeostatic synaptic scaling may also contribute to adult cortical plasticity (Turrigiano and Nelson, 2004). A recent study demonstrates that activity-dependent synaptic scaling in mouse visual cortex persists into adulthood (Goel and Lee, 2007). The dependence of aODP on the activity of the dominant, contralateral-eye input, as indicated by the asymmetry of MD and the plasticity after $\mathrm{BD}$, suggests further that one should look to compensatory mechanisms like synaptic scaling, rather than to synapse-specific plasticity mechanisms, for its explanation.

\section{Is adult cortical plasticity universal?}

Comparing experience-dependent plasticity in rodent visual cortex with that in somatosensory cortex reveals remarkable similarities between these processes (Fox and Wong, 2005). Characteristics such as a critical period for depression and a persistence of potentiation with a slower time course in adults may be common core features across different cortical areas. Functional plasticity is known to be extensive in adult life in auditory and somatosensory cortex from rodents to primates (Buonomano and Merzenich, 1998) and in the forebrain and even midbrain of birds (Knudsen, 2002; Tumer and Brainard, 2007). Functional OD plasticity after the closure of the critical period for ocular dominance columns as defined by the thalamocortical input to layer 4 has long been known, even in the animals held up as examples of a rigid critical period, such as cats and monkeys (LeVay et al., 1980; Daw et al., 1992). In few cases do we know the anatomical changes that mediate the widespread functional plasticity in adults, or even the extent to which the functional plasticity represents rewiring as opposed to changes in the strengths of existing connections. Recent longitudinal observations of the plasticity of anatomical connections in adult cortex span a wide range, from apparent rigidity to extensive plasticity (Grutzendler et al., 2002; Trachtenberg et al., 2002; Lee et al., 2006). Full elucidation of adult plasticity mechanisms at the cellular and molecular levels is also lacking. The further study of adult plasticity will be important for aiding recovery from cortical injury as well as, potentially, for enhancing learning capacity in adulthood.

Note added in proof. Evidence that ocular dominance plasticity declines further at ages greater than those studied here has recently been provided by Lehmann and Löwel (2008). Even $14 \mathrm{~d}$ of MD produced no significant plasticity after 7 months of age.

\section{References}

Antonini A, Fagiolini M, Stryker MP (1999) Anatomical correlates of functional plasticity in mouse visual cortex. J Neurosci 19:4388-4406.

Buonomano DV, Merzenich MM (1998) Cortical plasticity: from synapses to maps. Annu Rev Neurosci 21:149-186.

Cang J, Kalatsky VA, Löwel S, Stryker MP (2005) Optical imaging of the intrinsic signal as a measure of cortical plasticity in the mouse. Vis Neurosci 22:685-691.

Daw NW, Fox K, Sato H, Czepita D (1992) Critical period for monocular deprivation in the cat visual cortex. J Neurophysiol 67:197-202.

Fischer QS, Graves A, Evans S, Lickey ME, Pham TA (2007) Monocular 
deprivation in adult mice alters visual acuity and single-unit activity. Learn Mem 14:277-286.

Fox K, Wong RO (2005) A comparison of experience-dependent plasticity in the visual and somatosensory systems. Neuron 48:465-477.

Frenkel MY, Bear MF (2004) How monocular deprivation shifts ocular dominance in visual cortex of young mice. Neuron 44:917-923.

Frenkel MY, Sawtell NB, Diogo AC, Yoon B, Neve RL, Bear MF (2006) Instructive effect of visual experience in mouse visual cortex. Neuron 51:339-349.

Glazewski S, Fox K (1996) Time course of experience-dependent synaptic potentiation and depression in barrel cortex of adolescent rats. J Neurophysiol 75:1714-1729.

Goel A, Lee HK (2007) Persistence of experience-induced homeostatic synaptic plasticity through adulthood in superficial layers of mouse visual cortex. J Neurosci 27:6692-6700.

Gordon JA, Stryker MP (1996) Experience-dependent plasticity of binocular responses in the primary visual cortex of the mouse. J Neurosci 16:3274-3286.

Grutzendler J, Kasthuri N, Gan WB (2002) Long-term dendritic spine stability in the adult cortex. Nature 420:812-816.

Hofer SB, Mrsic-Flogel TD, Bonhoeffer T, Hübener M (2006a) Lifelong learning: ocular dominance plasticity in mouse visual cortex. Curr Opin Neurobiol 16:451-459.

Hofer SB, Mrsic-Flogel TD, Bonhoeffer T, Hübener M (2006b) Prior experience enhances plasticity in adult visual cortex. Nat Neurosci 9:127-132.

Jacobson SG, Mohindra I, Held R (1981) Development of visual acuity in infants with congenital cataracts. Br J Ophthalmol 65:727-735.

Kalatsky VA, Stryker MP (2003) New paradigm for optical imaging: temporally encoded maps of intrinsic signal. Neuron 38:529-545.

Karmarkar UR, Dan Y (2006) Experience-dependent plasticity in adult visual cortex. Neuron 52:577-585.

Kleinschmidt A, Bear MF, Singer W (1987) Blockade of "NMDA" receptors disrupts experience-dependent plasticity of kitten striate cortex. Science 238:355-358.

Knudsen EI (2002) Instructed learning in the auditory localization pathway of the barn owl. Nature 417:322-328.

Lee WC, Huang H, Feng G, Sanes JR, Brown EN, So PT, Nedivi E (2006) Dynamic remodeling of dendritic arbors in GABAergic interneurons of adult visual cortex. PLoS Biol 4:e29.

Lehmann K, Löwel S (2008) Age-dependent ocular dominance plasticity in adult mice. PLoS ONE 3:e3120.

LeVay S, Wiesel TN, Hubel DH (1980) The development of ocular domi- nance columns in normal and visually deprived monkeys. J Comp Neurol 191:1-51.

Mataga N, Fujishima S, Condie BG, Hensch TK (2001) Experiencedependent plasticity of mouse visual cortex in the absence of the neuronal activity-dependent marker egr1/zif268. J Neurosci 21:9724-9732.

Mioche L, Singer W (1989) Chronic recordings from single sites of kitten striate cortex during experience-dependent modifications of receptivefield properties. J Neurophysiol 62:185-197.

Pham TA, Graham SJ, Suzuki S, Barco A, Kandel ER, Gordon B, Lickey ME (2004) A semi-persistent adult ocular dominance plasticity in visual cortex is stabilized by activated CREB. Learn Mem 11:738-747.

Prusky GT, Alam NM, Beekman S, Douglas RM (2004) Rapid quantification of adult and developing mouse spatial vision using a virtual optomotor system. Invest Ophthalmol Vis Sci 45:4611-4616.

Prusky GT, Alam NM, Douglas RM (2006) Enhancement of vision by monocular deprivation in adult mice. J Neurosci 26:11554-11561.

Sale A, Maya Vetencourt JF, Medini P, Cenni MC, Baroncelli L, De Pasquale R, Maffei L (2007) Environmental enrichment in adulthood promotes amblyopia recovery through a reduction of intracortical inhibition. Nat Neurosci 10:679-681.

Sawtell NB, Frenkel MY, Philpot BD, Nakazawa K, Tonegawa S, Bear MF (2003) NMDA receptor-dependent ocular dominance plasticity in adult visual cortex. Neuron 38:977-985.

Tagawa Y, Kanold PO, Majdan M, Shatz CJ (2005) Multiple periods of functional ocular dominance plasticity in mouse visual cortex. Nat Neurosci 8:380-388.

Trachtenberg JT, Chen BE, Knott GW, Feng G, Sanes JR, Welker E, Svoboda $\mathrm{K}$ (2002) Long-term in vivo imaging of experience-dependent synaptic plasticity in adult cortex. Nature 420:788-794.

Tumer EC, Brainard MS (2007) Performance variability enables adaptive plasticity of "crystallized" adult birdsong. Nature 450:1240-1244.

Turrigiano GG, Nelson SB (2004) Homeostatic plasticity in the developing nervous system. Nat Rev Neurosci 5:97-107.

Villarreal DM, Do V, Haddad E, Derrick BE (2002) NMDA receptor antagonists sustain LTP and spatial memory: active processes mediate LTP decay. Nat Neurosci 5:48-52.

Wiesel TN (1982) Postnatal development of the visual cortex and the influence of environment. Nature 299:583-591.

Yoshimura Y, Ohmura T, Komatsu Y (2003) Two forms of synaptic plasticity with distinct dependence on age, experience, and NMDA receptor subtype in rat visual cortex. J Neurosci 23:6557-6566. 